\title{
A theoretical model on pore size distribution in low dielectric constant nanoporous silica films
}

\author{
Jyh-Ping Hsu*, Shih-Hsing Hung, Wen-Chang Chen \\ Department of Chemical Engineering, National Taiwan University, Taipei 10617, Taiwan
}

Received 30 August 2003; received in revised form 1 March 2004; accepted 1 March 2004

\begin{abstract}
A pore growth mechanism is proposed for the description of the pore size distribution and the porosity of the low dielectric constant materials. A theoretical model is constructed to portray the evolution of pore size distribution in nanoporous silica. The applicability of the model is justified by fitting it to the experimental data reported in the literature, and the effects of the essential parameters of the model on both the pore size distribution and on the porosity of a silica film are examined. The results of numerical simulation reveal that the quality of a film can be improved by increasing the rate of production of pore seeds. In particular, the total number of pores increases with the increase in the rate of production of pore seeds, and the faster the growth rate of pore the greater the number of large pores and the larger the total number of pores produced.
\end{abstract}

(C) 2004 Elsevier B.V. All rights reserved.

PACS: 73.61.P; 73.50.Yg

Keywords: Dielectrics; Growth mechanism; Nanoporous silica film; Pore size distribution

\section{Introduction}

Modern electronic devices are characterized by light, thin, short, small in appearance, high speed, and efficiency in operation. To achieve these requirements, key issues such as interconnect RC delay, power consumption, and crosstalk between adjacent electronic elements become significant. One possible solution is to increase the number of metallization layers. This, however, requires significant costs and is reaching limitations in performance gain as the number of layers exceeds 5-7 [1]. Another way is to use metals of high conductivity and/or materials with a low dielectric constant $(k)$ [2]. In particular, the development of low $\mathrm{k}$ materials has become one of the most popular subjects in semiconductor industries [3].

The low $k$ materials developed recently can be classified into several categories: spin-on-glass (SOG) materials, organic polymers, organic-inorganic hybrid materials, and porous materials [3]. SOG materials such as hydrogen silsesquioxane (HSQ) and methyl silsesquioxane (MSQ)

\footnotetext{
* Corresponding author. Tel.: +886-2-23637448; fax: +886-223623040

E-mail address: jphsu@ntu.edu.tw (J.-P. Hsu).
}

have a dielectric constant in the range of 2.5-3.0. The structural transformation, electrical, thermal, mechanical properties, and their integration with integrated circuit devices have been reported [3]. Low dielectric constant organic polymers generally contain aromatic or fluorine structure, such as fluoropolyimide, parylene, Teflon AF, and Flare. These materials may have a dielectric constant in the range of 1.9-3.0. However, their thermal stability is mostly lower than $400{ }^{\circ} \mathrm{C}$ [3-8]. Hybrid materials of MSQ and polyimide have been tested to improve the thermal and mechanical properties of polyimide [3]. However, their dielectric constants generally exceed 2.0 , and, therefore, might be unsuitable for the integrated circuit process with a line-width of nanosized scale. Nanoporous materials, for example, nanoporous silica, are possible candidates for the low $k$ materials, which have a dielectric constant smaller than 2.0. In addition to the low dielectric constant, they also have the merits such as high thermal stability and processibility [1-3].

Nanoporous silica is a material with many nanopores distributed in silica. According to Jin et al. [2], its dielectric constant is a linear combination of air (dielectric constant about unity) and dense silica. That is, the dielectric constant is linearly dependent on its porosity; the greater the porosity, 
the smaller the dielectric constant. However, the strength of a silica film decreases as its porosity increases. Also, to avoid circuit defects, it is required that the pore size of a silica film should be smaller than the circuit feature size. This implies that the control of both pore size and its distribution plays the key role in the development of low $k$ nanoporous silica. The distribution of pore size in a silica film depends on several factors. These include, for example, the diffusion of solvent inside the silica film, the mass transfer at the solvent-gas interface, the concentration of solvent, the curing temperature, and the nitrogen-blowing rate. Unfortunately, relevant discussions about the evolution of pore size distribution during silica film processing are limited in the literature, and its mechanism is still not well understood. In this study, a pore growth mechanism is proposed, and a theoretical model is derived to describe the evolution of pore size distribution in nanoporous silica. The essential parameters affecting the pore size distribution in a silica film are discussed.

\section{Modeling}

Nanoporous silica is generally prepared by various alkoxide precursors under acid or base catalysis, followed by deposition, evaporation, and drying. The experimental system of the present modeling is based on that of Smith et al. [4,9]. Smith et al. [4] used a two-step acid-base catalysis procedure for preparing the precursor solution for nanoporous silica films. In the first step, a tetraethyl orthosilicate solution was prepared by refluxing tetraethoxy silane (TEOS), ethanol, deionized water and hydrochloric acid (in a molar ratio of $1: 3: 1: 7.3 \times 10^{-4}$ ) for $1.5 \mathrm{~h}$ at $70{ }^{\circ} \mathrm{C}$. In the second step, the stock solution was diluted to 70 vol.\% with ethanol. Then, $1 \mathrm{ml}$ of the catalyst $\left(0.5 \mathrm{M} \mathrm{NH}_{4} \mathrm{OH}\right)$ was added to $10 \mathrm{ml}$ of the stock solution. The gelation reaction of the above solution was around $20 \mathrm{~min}$. In the following, the solution was deposited on a silica wafer, followed by evaporation and dried to obtain a porous silica film. A schematic representation of our model system is illustrated in Fig. 1 where the silica gel film on a wafer surface is heated from below. The evaporation of the solvent (ethanol) produces pore seeds of a negligible size. The so-called pore

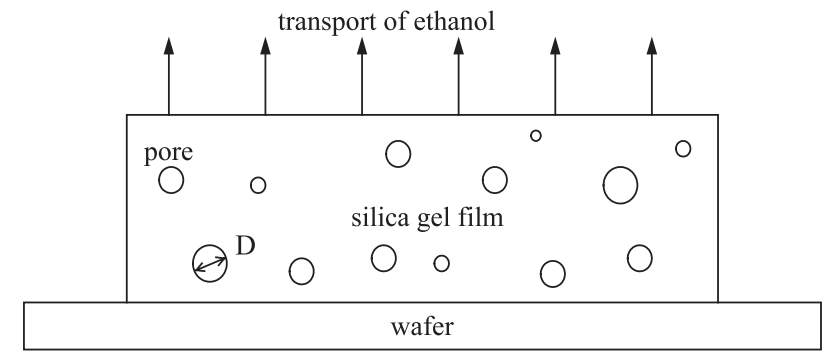

Fig. 1. Schematic representation of the problem under consideration. The evaporation of ethanol inside the silica gel film generates pore seeds, some of which grow with time to yield larger pores. seed is the space produced when solvent evaporates. Because physical phenomena usually occur continuously this space may contain few solvent atoms, and therefore, assuming that its size is negligible is realistic. The growth of pore seeds at the consumption of evaporated ethanol with curing time yields pores of various sizes. The evaporation and the subsequent transport of the ethanol immediately near the film-gas interface to the bulk gas phase do not contribute to the production of pore seeds. We assume that the rate of production of pore seeds is a function of the molar fraction of ethanol inside the film. At each stage, only a fraction $f$ of all the pores will grow, and the rests remain unchanged. The rate of ethanol evaporated directly from the film-gas interface to the bulk gas phase is a function of the molar fraction of ethanol inside the film. Since the amounts of $\mathrm{HCl}$ and $\mathrm{NH}_{4} \mathrm{OH}$ are negligible in comparison with other components [4], the number of moles of silica in the silica gel film at the end of curing process is essentially that of TEOS initially. On the basis of these statements, the rate of production of pore seeds inside the film can be expressed as

$\frac{\mathrm{d} N_{0}(t)}{\mathrm{d} t}=k_{\mathrm{p}}[X(t)]$

where $N_{0}(t)$ is the number of pore seeds produced at time $t$, $X(t)=n_{\mathrm{EtOH}}(t) /\left(n_{\mathrm{TEOS}}^{0}+n_{\mathrm{EtOH}}(t)\right)$ is the molar fraction of ethanol at time $t, n_{\mathrm{EtOH}}(t)$ and $n^{0}{ }_{\mathrm{TEOS}}$ being respectively the number of moles of ethanol at time $t$ and initially, and $k_{\mathrm{p}}$ is a rate constant. We assume that the growth rate of a pore is linearly dependent upon its size, that is,

$\frac{\mathrm{d} D(t)}{\mathrm{d} t}=a(1+b D(t))$

where $D(t)$ is the diameter of a pore at time $t$, and $a$ and $b$ are adjustable parameters, which need to be estimated from experimental data. Suppose that the rate of evaporation of ethanol from the film-gas interface to the bulk gas phase can be expressed by

$\frac{\mathrm{d} n^{\mathrm{ev}}}{\mathrm{d} t}=k_{\mathrm{ev}} X(t)$

where $n^{\text {ev }}$ is the number of moles of ethanol evaporated from the film-gas interface to the bulk gas phase, and $k_{\mathrm{ev}}$ is a constant.

\subsection{Numerical procedure}

For convenience, the time scale is discretized as $t=i \Delta t$, $i=0,1,2, \ldots, I, \ldots, I I$, where $I I \Delta t$ denotes the curing time. Because the evaporation of solvent is of stochastic nature microscopically, a pore may grow to become a larger one in a certain time interval, or it may remain the same size (i.e., not grow) at that time interval, therefore, we assume that for each increment of time $\Delta t$ in this time interval a fraction $f$ of the pores of various sizes will grow, and the rests remain 
unchanged. According to Eq. (2), the diameter of a pore after a growth time of $k \Delta t$ becomes

$D(k)=\frac{1}{b}\left[\mathrm{e}^{a b k \Delta t}-1\right]$

$k=1,2, \ldots, I-1$ at time $I \Delta t$. The total number of pore seeds at time $I \Delta t, N_{I}(0)$, includes those newly produced in the time
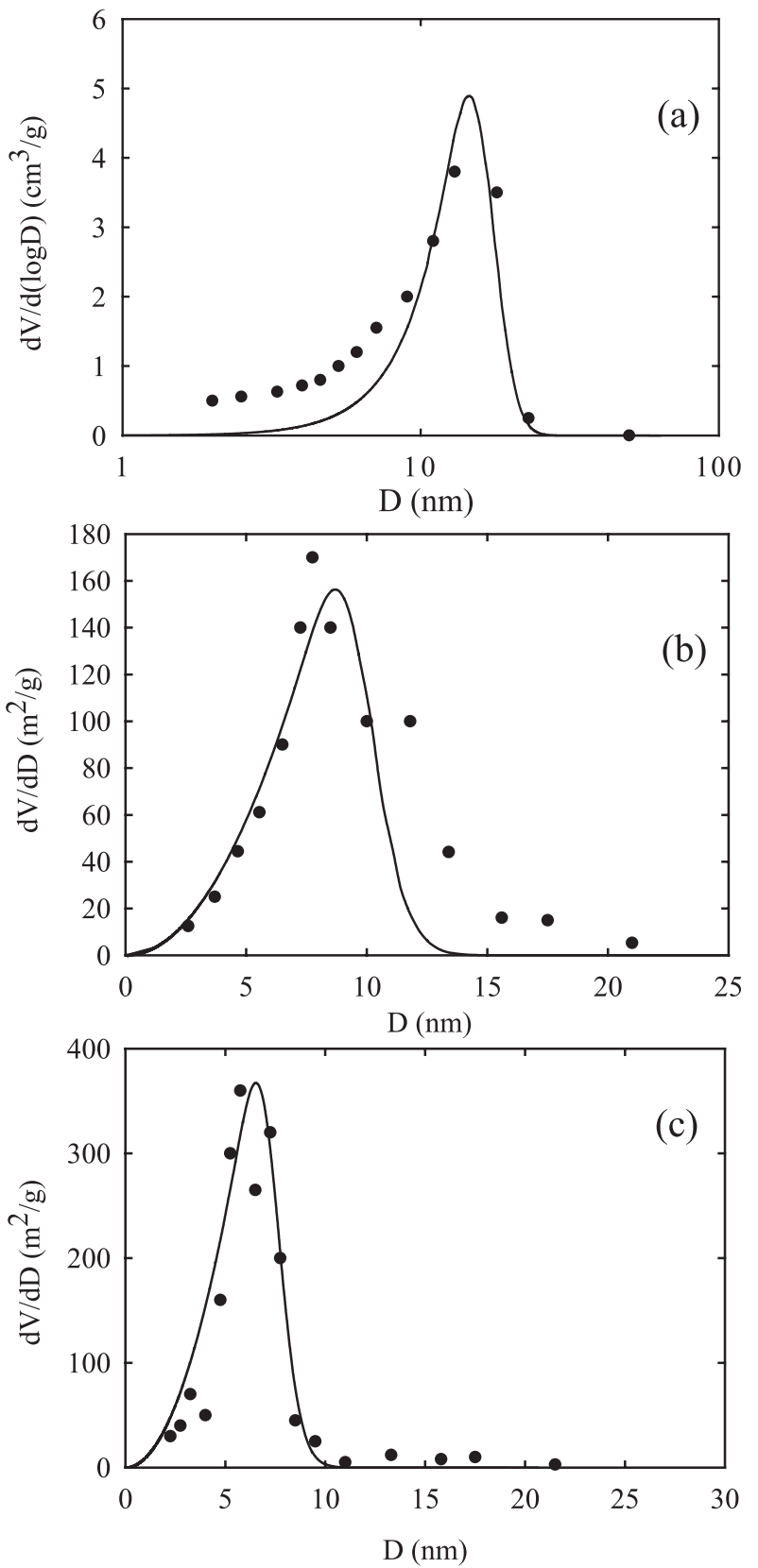

Fig. 2. Pore size distribution where solid curves represent the results based on present model. (a) Experimental data of Smith et al. [4]. Fitted parameters are $k_{\mathrm{p}}=1 \times 10^{19} \mathrm{~s}^{-1}, a=2.5 \times 10^{-10} \mathrm{~m} / \mathrm{s}$, and $b=5 \times 10^{10} \mathrm{~m}^{-1}$. (b) Experimental data of Ramos et al. [9]. Fitted parameters are $k_{\mathrm{p}}=7 \times 10^{18} \mathrm{~s}^{-1}$, $a=2 \times 10^{-10} \mathrm{~m} / \mathrm{s}$, and $b=4 \times 10^{10} \mathrm{~m}^{-1}$. (c) Experimental data of Ramos et al. [9]. Fitted parameters are $k_{\mathrm{p}}=3 \times 10^{19} \mathrm{~s}^{-1}, a=2.2 \times 10^{-10} \mathrm{~m} / \mathrm{s}$, and $b=3.75 \times 10^{10} \mathrm{~m}^{-1}$. Other parameters: $n_{\mathrm{TEOS}}=1 / 60 \mathrm{~mol}, \Delta t=0.0002 \mathrm{~s}$, $\rho=0.789 \mathrm{~g} \mathrm{ml}^{-1}, \rho_{\mathrm{SiO}_{2}}=2.26 \mathrm{~g} \mathrm{ml}^{-1}, f=0.18, k_{\mathrm{ev}}=0.07 \mathrm{~mol} / \mathrm{s}$, and $n_{\mathrm{EtOH}}^{0}=$ $0.2 \mathrm{~mol}$.
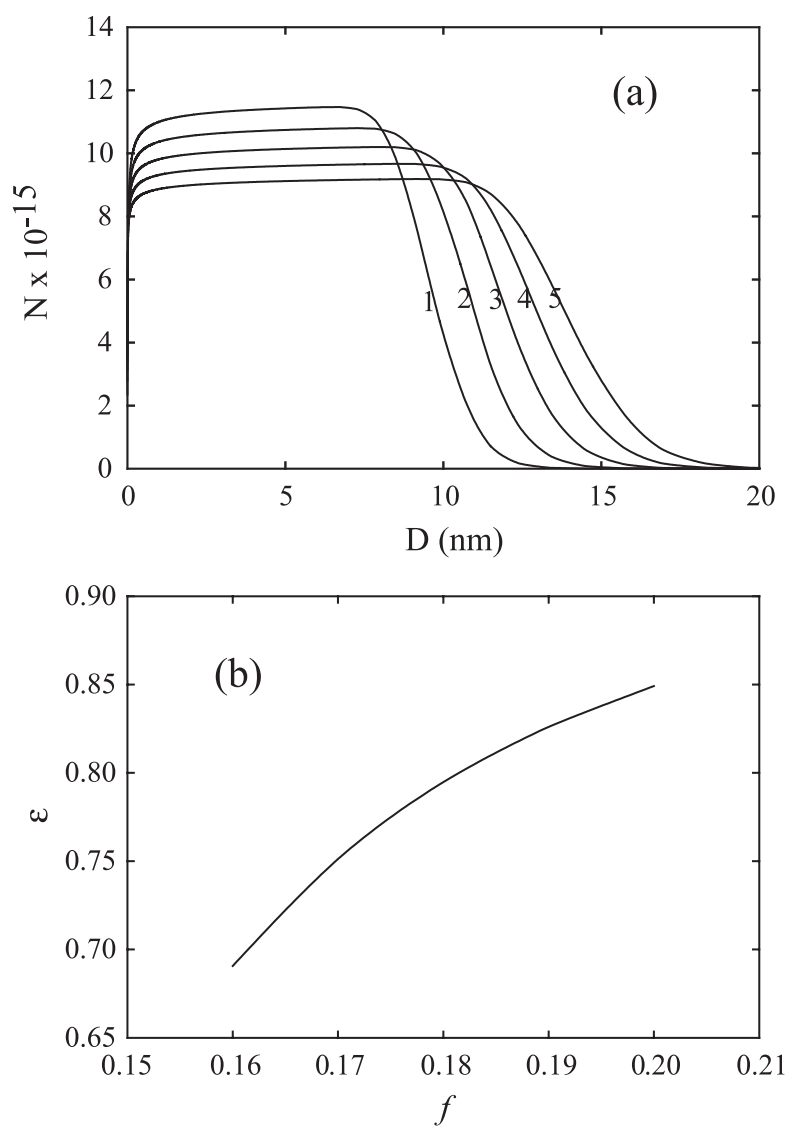

Fig. 3. (a) Pore size distribution at various $f$ for the case when $k_{\mathrm{p}}=1 \times 10^{19}$ $\mathrm{s}^{-1}, a=2.25 \times 10^{-10} \mathrm{~m} / \mathrm{s}, b=4.5 \times 10^{10} \mathrm{~m}^{-1}, k_{\mathrm{ev}}=0.07 \mathrm{~mol} / \mathrm{s}$, and $n_{\mathrm{EtOH}}^{0}=0.2$ mol. Curve $1, f=0.16 ; 2, f=0.17 ; 3, f=0.18 ; 4, f=0.19 ; 5, f=0.2$. (b) The corresponding variation in the porosity of silica gel film $\varepsilon$ as a function of $f$. Other parameters are the same as those of Fig. 2, except that $f$ varies.

interval from $(I-1) \Delta t$ to $I \Delta t$, and those produced before $(I-1) \Delta t$ but did not grow during that time interval. Therefore, we have

$N_{I}(0)=k_{\mathrm{p}} X_{I-1} \Delta t+(1-f) N_{I-1}(0)$

where $X_{I-1}$ is the molar fraction of ethanol at time $(I-1) \Delta t$. The total number of pores with diameter $D(k)$ at time $I \Delta t$, $N_{I}(k)$, includes those with diameter $D(k)$ at time $(I-1) \Delta t$ and not grow during the time interval from $(I-1) \Delta t$ to $I \Delta t$, and those with diameter $D(k-1)$ at time $(I-1) \Delta t$ and grow during the time interval. Therefore, we have

$N_{I}(k)=(1-f) N_{I-1}(k)+f N_{I-1}(k-1)$

If the pores are spherical, then the total volume of pores, $V_{I}$, at time $I \Delta t$ is

$V_{I}=\sum_{k=1}^{I-1} \frac{1}{6} \pi D(k)^{3} N_{I}(k)$ 

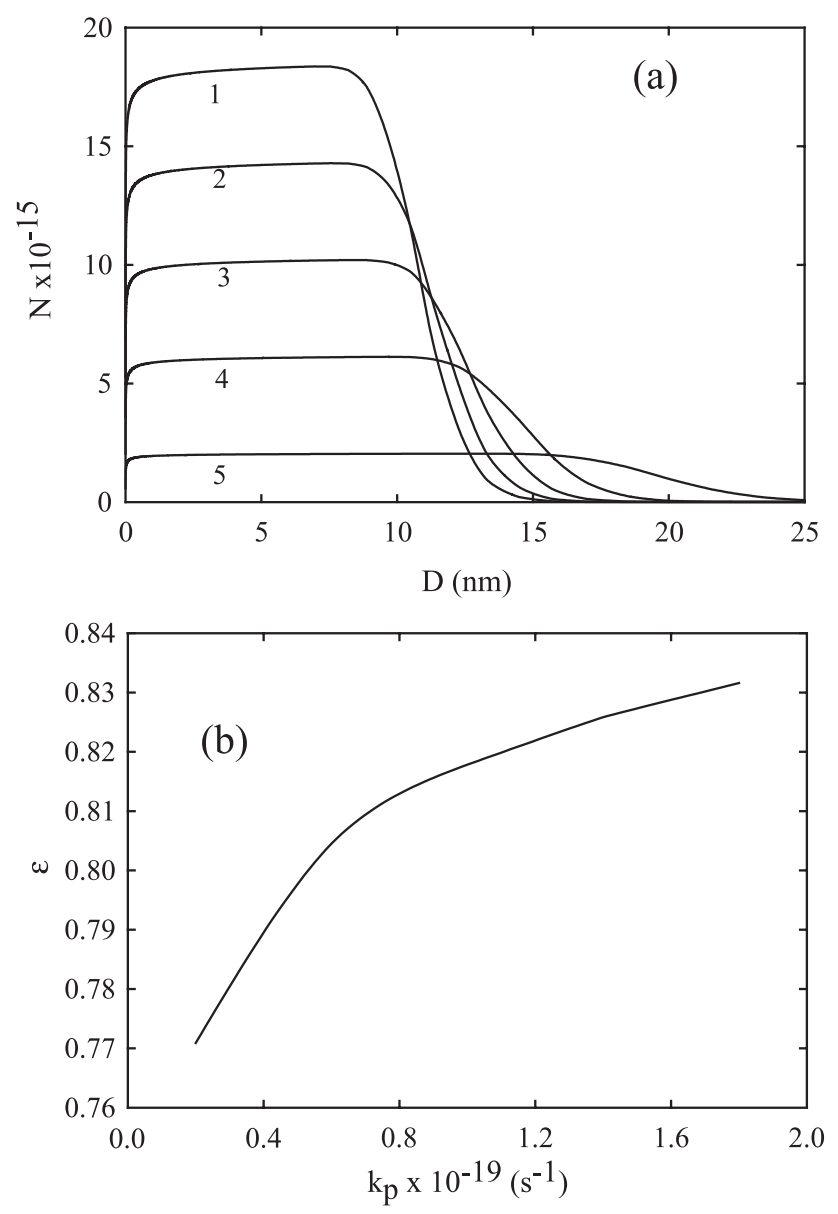

Fig. 4. (a) Pore size distribution at various $k_{\mathrm{p}}$ for the case when $f=0.18$, $a=2.25 \times 10^{-10} \mathrm{~m} / \mathrm{s}, b=4.5 \times 10^{10} \mathrm{~m}^{-1}, k_{\mathrm{ev}}=0.07 \mathrm{~mol} / \mathrm{s}$, and $n_{\mathrm{EtOH}}^{0}=0.2 \mathrm{~mol}$. Curve $1, k_{\mathrm{p}}=1.8 \times 10^{19} \mathrm{~s}^{-1} ; 2, k_{\mathrm{p}}=1.4 \times 10^{19} \mathrm{~s}^{-1} ; 3, k_{\mathrm{p}}=1.0 \times 10^{19} \mathrm{~s}^{-1} ; 4$, $k_{\mathrm{p}}=0.6 \times 10^{19} \mathrm{~s}^{-1} ; 5, k_{\mathrm{p}}=0.2 \times 10^{19} \mathrm{~s}^{-1}$. (b) The corresponding variation in the porosity of silica gel film $\varepsilon$ as a function of $k_{\mathrm{p}}$. Other parameters are the same as those of Fig. 2, except that $k_{\mathrm{p}}$ varies.

Integrating Eq. (3) from $t=0$ to $t=I \Delta t$ yields the total number of moles of ethanol evaporated from film-gas interface to the bulk gas phase. We have

$n_{I}^{\mathrm{ev}}=\sum_{i=1}^{I} k_{\mathrm{ev}} X_{i-1} \Delta t$

The number of moles of ethanol inside a silica film at time $I \Delta t$ equals the initial number of moles of ethanol minus the number of moles of ethanol leaving directly from the filmgas interface to the bulk gas phase and the number of moles of ethanol which becomes pores. We have

$n_{I, \mathrm{EtOH}}=n_{\mathrm{EtOH}}^{0}-n_{I}^{\mathrm{ev}}-V_{I} \rho$

where $N_{\mathrm{EtOH}}^{0}$ is the initial number of moles of ethanol, and $\rho$ is the ethanol density. Then, we can calculate new $X_{I}$ and then repeat these steps until the ethanol is used up. Then, the porosity of the silica gel film, $\varepsilon$, can be expressed as

$\varepsilon=\frac{V_{I}}{V_{I}+V_{\mathrm{SiO}_{2}}}=\frac{V_{I}}{V_{I}+\left(n_{\mathrm{TEOS}} / \rho_{\mathrm{SiO}_{2}}\right)}$ where $V_{\mathrm{SiO}_{2}}$ is the dense volume of silica, and $\rho_{\mathrm{SiO}_{2}}$ is the density of $\mathrm{SiO}_{2}$. Note that the number of moles of $\mathrm{SiO}_{2}$ equals that of TEOS $\left(n_{\mathrm{TEOS}}\right)$.

\section{Results and discussions}

The applicability of the present model is examined by fitting it to the available data in the literature [4,9]. Fig. 2 shows the experimental data for the pore size distribution of nanoporous silica film [4,9] and the results predicted by the present model. Solid curves represent the results based on the present model that assume the rate of growth of a pore is dependent of its size. In the data fitting procedure, $1.0 \mathrm{~g}$ of nanoporous silica was chosen as a basis, which is equivalent to $0.0167 \mathrm{~mol} \mathrm{SiO}_{2}$. The number of moles of silica in the silica gel film at the end of curing process is equal to that of TEOS initially, $0.0167 \mathrm{~mol}$. The density of ethanol, $\rho$, and that of $\mathrm{SiO}_{2}$, $\rho_{\mathrm{SiO}_{2}}$, are 0.789 and $2.26 \mathrm{~g} \mathrm{ml}^{-1}$, respectively. According to the experimental procedure described in the Modeling,
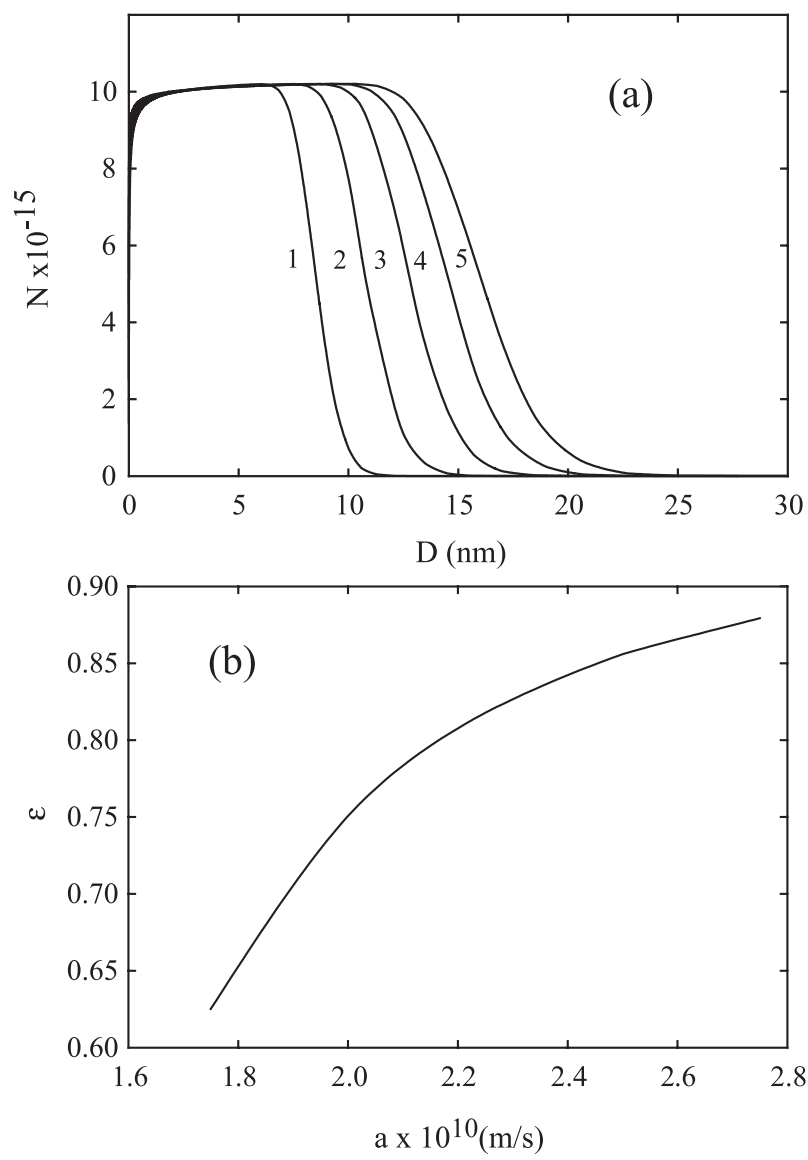

Fig. 5. (a) Pore size distribution at various $a$ for the case when $f=0.18$, $k_{\mathrm{p}}=1 \times 10^{19} \mathrm{~s}^{-1}, b=4.5 \times 10^{10} \mathrm{~m}^{-1}, k_{\mathrm{ev}}=0.07 \mathrm{~mol} / \mathrm{s}$, and $n_{\mathrm{EtOH}}^{0}=0.2 \mathrm{~mol}$. Curve $1, a=1.75 \times 10^{-10} \mathrm{~m} / \mathrm{s} ; 2, a=2 \times 10^{-10} \mathrm{~m} / \mathrm{s} ; 3, a=2.25 \times 10^{-10} \mathrm{~m} / \mathrm{s} ; 4$, $a=2.5 \times 10^{-9} \mathrm{~m} / \mathrm{s} ; 5, a=2.75 \times 10^{-10} \mathrm{~m} / \mathrm{s}$. (b) The corresponding variation in the porosity of silica gel film $\varepsilon$ as a function of $a$. Other parameters are the same as those of Fig. 2, except that $a$ varies. 
the ratio of the number of moles of ethanol and that of TEOS is about 12. The value of $f$ and the rate constant of evaporation, $k_{\mathrm{ev}}$, are estimated as 0.18 and $0.07 \mathrm{~mol} / \mathrm{s}$, respectively. Fig. 2 reveals that the performance of the present model is satisfactory.

The simulated pore size distribution and the corresponding porosity of a silica gel film $\varepsilon$ at various $f$ are shown in Fig. 3. Fig. 3a indicates that a larger $f$ yields a smaller number of small pores and a larger number of large pores. This is expected because a large $f$ implies that a large number of pores will grow. Also, since a large amount of ethanol is consumed, the rate of generation of pore seeds decreases. Fig. $3 \mathrm{~b}$ reveals that $\varepsilon$ increases with the increase in $f$. This suggests that the porosity of a silica film can be controlled by $f$, which, in turn, is controlled by curing conditions. Note that the relation between $\varepsilon$ and $f$ is of nonlinear nature.

The simulated pore size distribution and the corresponding porosity of a silica gel film $\varepsilon$ at various $k_{\mathrm{p}}$ are shown in Fig. 4. As can be seen in Fig. $4 \mathrm{a}$, the larger the $k_{\mathrm{p}}$, the greater the number of small pores and the smaller the
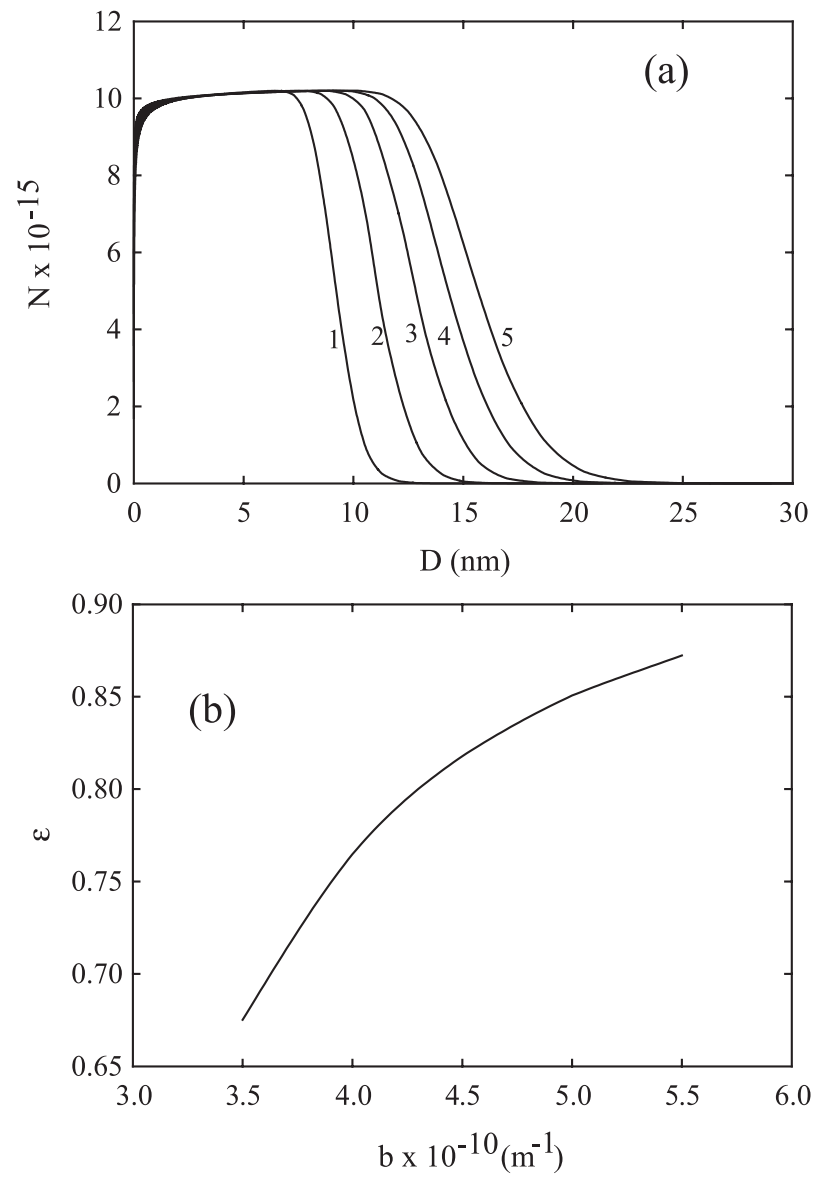

Fig. 6. (a) Pore size distribution at various $b$ for the case when $f=0.18$, $k_{\mathrm{p}}=1 \times 10^{19} \mathrm{~s}^{-1}, a=2.25 \times 10^{-10} \mathrm{~m} / \mathrm{s}, k_{\mathrm{ev}}=0.07 \mathrm{~mol} / \mathrm{s}$, and $n_{\mathrm{EtOH}}^{0}=0.2 \mathrm{~mol}$. Curve $1, b=3.5 \times 10^{10} \mathrm{~m}^{-1} ; 2, b=4 \times 10^{10} \mathrm{~m}^{-1} ; 3, b=4.5 \times 10^{10} \mathrm{~m}^{-1} ; 4$, $b=5 \times 10^{10} \mathrm{~m}^{-1} ; 5, b=5.5 \times 10^{10} \mathrm{~m}^{-1}$. (b) The corresponding variation in the porosity of silica gel film $\varepsilon$ as a function of $b$. Other parameters are the same as those of Fig. 2, except that $b$ varies.
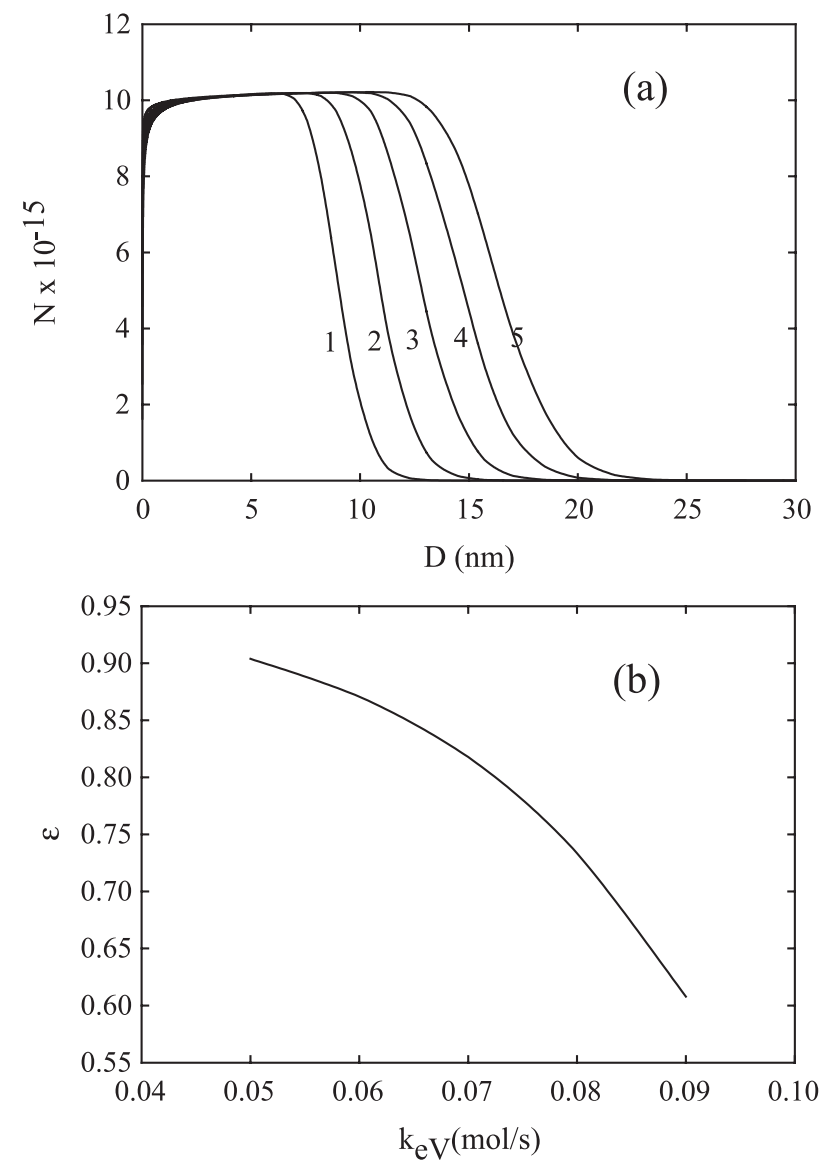

Fig. 7. (a) Pore size distribution at various $k_{\mathrm{ev}}$ for the case when $f=0.18$, $k_{\mathrm{p}}=1 \times 10^{19} \mathrm{~s}^{-1}, a=2.25 \times 10^{-10} \mathrm{~m} / \mathrm{s}, b=4.5 \times 10^{10} \mathrm{~m}^{-1}$, and $n_{\mathrm{EtOH}}^{0}=0.2 \mathrm{~mol}$. Curve $1, k_{\mathrm{ev}}=0.09 \mathrm{~mol} / \mathrm{s} ; 2, k_{\mathrm{ev}}=0.08 \mathrm{~mol} / \mathrm{s} ; 3, k_{\mathrm{ev}}=0.07 \mathrm{~mol} / \mathrm{s} ; 4, k_{\mathrm{ev}}=0.06$ $\mathrm{mol} / \mathrm{s} ; 5, k_{\mathrm{ev}}=0.05 \mathrm{~mol} / \mathrm{s}$. (b) The corresponding variation in the porosity of silica gel film as a function of $k_{\mathrm{ev}}$. Other parameters are the same as those of Fig. 2, except that $k_{\text {ev }}$ varies.

number of large pores. Also, the total number of pores increases with the increase in $k_{\mathrm{p}}$. This is because the larger the $k_{\mathrm{p}}$ the faster the rate of production of pore seeds. According to Fig. $4 \mathrm{~b}, \varepsilon$ increases with the increase in $k_{\mathrm{p}}$, and they are nonlinearly related. Fig. 4 implies that the quality of a film can be improved by increasing the value of $k_{\mathrm{p}}$, because the larger the $k_{\mathrm{p}}$ the narrower the pore size distribution and the larger the porosity.

Fig. 5 illustrates the simulated pore size distribution and the corresponding porosity of a silica gel film $\varepsilon$ at various $a$. Fig. 5a reveals that the larger the $a$ the greater the number of large pores and the greater the total number of pores produced. The former is because the larger the $a$, the faster the rate of growth of a pore. The latter is due to the fact that it is more probable for the evaporated ethanol to become part of a pore before it escapes to the bulk gas phase. As suggested by Fig. $5 \mathrm{~b}$, the larger the $a$ the greater the $\varepsilon$, and they are nonlinearly correlated.

Fig. 6 illustrates the simulated pore size distribution and the corresponding porosity of a silica gel film $\varepsilon$ at various $b$. Fig. 6 a indicates that the larger the $b$ the greater the number 

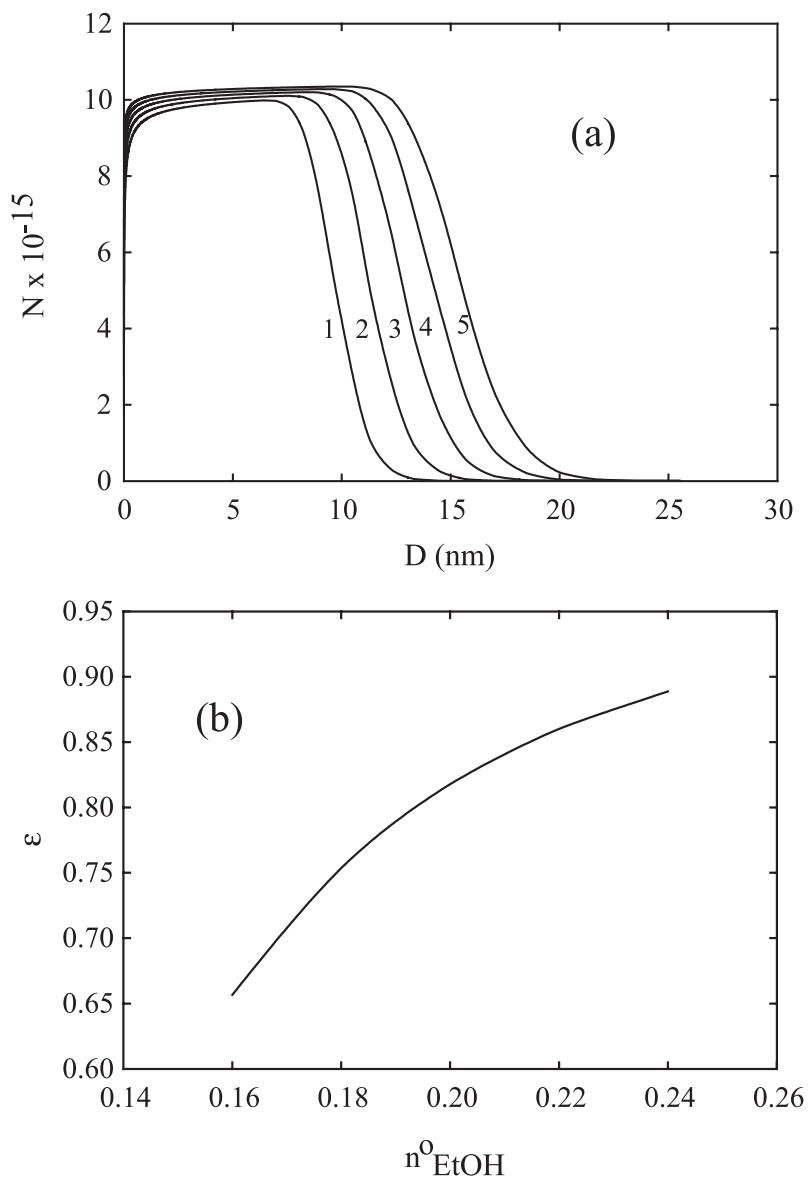

Fig. 8. (a) Pore size distribution at various $n_{\mathrm{EtOH}}^{0}$ for the case when $f=0.18$, $k_{\mathrm{p}}=1 \times 10^{19} \mathrm{~s}^{-1}, a=2.25 \times 10^{-10} \mathrm{~m} / \mathrm{s}, \quad b=4.5 \times 10^{10} \mathrm{~m}^{-1}, k_{\mathrm{ev}}=0.07 \mathrm{~mol} / \mathrm{s}$. Curve $1, n_{\mathrm{EtOH}}^{0}=0.16 \mathrm{~mol} ; 2, n_{\mathrm{EtOH}}^{0}=0.18 \mathrm{~mol} ; 3, n_{\mathrm{EtOH}}^{0}=0.2 \mathrm{~mol} ; 4$, $n_{\mathrm{EtOH}}^{0}=0.22 \mathrm{~mol} ; 5, n_{\mathrm{EtOH}}^{0}=0.24 \mathrm{~mol}$. (b) The corresponding variation in the porosity of silica gel film $\varepsilon$ as a function of $n_{\mathrm{EtOH}}^{0}$. Other parameters are the same as those of Fig. 2, except that $n_{\mathrm{EtOH}}^{0}$ varies.

of large pores and the larger the total number of pores produced. The former is because a large $b$ implies a fast rate of growth of a pore, as described by Eq. (2). The latter arises from that it is more probable for the evaporated ethanol to become part of a pore before it escapes to the bulk gas phase. As suggested by Fig. 6b, the larger the $b$ the greater the $\varepsilon$, and they are nonlinearly correlated.

Fig. 7 shows the simulated pore size distribution and the corresponding porosity of a silica gel film $\varepsilon$ at various $k_{\mathrm{ev}}$. Fig. 7a reveals that the larger the $k_{\mathrm{ev}}$ the smaller the total number of pores. This is expected because a larger $k_{\mathrm{ev}}$ implies a faster rate of ethanol leaving directly from the film-gas interface to the bulk gas phase, and the smaller the amount of ethanol available for the generation of pores. As can be seen in Fig. $7 \mathrm{~b}$, the larger the $k_{\mathrm{ev}}$ the smaller the porosity of a film, which is expected.

The simulated pore size distribution and the corresponding porosity of a silica gel film $\varepsilon$ at various $n_{\mathrm{EtOH}}^{0}$ are presented in Fig. 8. Fig. 8a reveals that both the total number of pores and the number of pores of each size increase with the increase in $n_{\mathrm{EtOH}}^{0}$. These are expected because a large $n_{\mathrm{EtOH}}^{0}$ implies a large amount of ethanol is available for both the generation of pore seeds and the growth of pores. As can be seen in Fig. 8b, $\varepsilon$ increases with the increase in $n_{\mathrm{EtOH}}^{0}$, and they are nonlinearly correlated.

\section{Conclusion}

A theoretical kinetic model is proposed for the description of the evolution of the pore size distribution of a nanoporous silica film. This model contains several key parameters, which are found to have a significant influence on both the pore size distribution and the porosity of a film. These include the rate of generation of pore seeds, the growth rate of pore, the fraction of the number of pore seeds that will grow, the rate of transport of organic solvent from the filmgas interface to the bulk gas phase, and the amount of organic solvent initially present in the film. The results obtained provide necessary information for both the design and the control of the curing step of semiconductor processing.

\section{Acknowledgements}

This work is supported by the National Science Council of the Republic of China.

\section{References}

[1] T. Ramos, K. Roderick, A. Maskara, D.M. Smith, in: K. Uram, H. Treichel, A. Lagendijk (Eds.), Low-Dielectric Constant Materials: II. Boston, MA, U.S.A., December 2-3, 1996, Materials Research Society Symposium Proceeding, vol. 443, 1997, p. 91.

[2] C. Jin, S. List, E. Zielinski, in: C. Chiang, J.T. Wetzel, T.-M. Lu, P.S. Ho (Eds.), Low-Dielectric Constant Materials: IV. San Francisco, CA, U.S.A., April 14-16, 1998, Materials Research Society Symposium Proceeding, vol. 511, 1998, p. 213.

[3] R.D. Miller, R. Beyers, K.R. Carter, R.F. Cook, M. Harbison, C.J. Hawker, J.H. Hedrick, V. Lee, E. Liniger, C. Nguyen, J. Remenar, M. Sherwood, M. Trollsas, W. Volksen, D.Y. Yoon, in: J. Hummel, K. Endo, W.W. Lee, M. Mills, S.-Q. Wang (Eds.), Low-Dielectric Constant Materials: V. San Francisco, CA, U.S.A., April 5-8, 1999, Materials Research Society Symposium Proceeding, vol. 565, 1999, p. 3.

[4] D.M. Smith, J. Anderson, C.C. Cho, G.P. Johnston, S.P. Jeng, in: T.-M. Lu, S.P. Murarka, T.S. Kuan, C.H. Ting (Eds.), Low-Dielectric Constant Materials-Synthesis and Applications in Microelectronics, San Francisco, CA, U.S.A., April 17-19, 1995, Materials Research Society Symposium Proceeding, vol. 381, 1995, p. 261.

[5] J.K. Hong, H.S. Yang, M.H. Jo, H.H. Park, S.Y. Choi, Thin Solid Film 308-309 (1997) 495.

[6] S. Baskaran, J. Liu, K. Domansky, N. Kohler, X. Li, C. Coyle, G.E. Fryxell, S. Thevuthasan, R.E. Williford, Adv. Mat. 12 (2000) 291.

[7] A.V. Rao, R.B. Wagh, D. Haranath, P.P. Risbud, S.D. Kumbhare, Ceram. Int. 25 (1999) 505.

[8] C.-C. Cho, D.M. Smith, J. Anderson, Mater. Chem. Phys. 42 (1995) 91.

[9] T. Ramos, K. Rhoderick, R. Roth, L. Brungardt, S. Wallace, J. Drage, J. Dunne, D. Endisch, R. Katsanes, N. Viernes, D.M. Smith, in: C. Chiang, J.T. Wetzel, T.-M. Lu, P.S. Ho (Eds.), Low-Dielectric Constant Materials: IV. San Francisco, CA, USA, April 14-16, 1998, Materials Research Society Symposium Proceeding, vol. 511, 1998, p. 105. 